Electroanalysis, 2007, Volume 19, Issue 4, Pages 487 - 495

\title{
Flexible Ultra-Thin PolyDVB/EVB Composite Membranes for the Optimisation of a Whole Blood Glucose Sensor
}

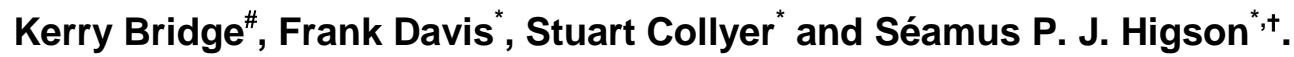

${ }^{*}$ Cranfield Health, Cranfield University, Silsoe, Beds, MK45 4DT, UK

\# Manchester Materials Science Centre, UMIST, Grosvenor Street, Manchester, M1 7HS. U.K.

${ }^{\dagger}$ Corresponding author. Fax (+44) 01525 863533, email s.p.j.higson@cranfield.ac.uk ABSTRACT

An ultrathin composite membrane has been developed as the outer covering barrier in a model amperometric glucose oxidase enzyme electrode. The membrane was formed by cathodic electropolymerisation of divinylbenzene/ethylvinylbenzene at the surface of a gold coated polyester support membrane. Permeability coefficients were determined for $\mathrm{O}_{2}$ and glucose across membranes with a range of polymer thicknesses. Anionic interferents (such as ascorbate), were screened from the working electrode via a charge exclusion mechanism. The enzyme electrode showed an initial $10 \%$ signal drift when first exposed to whole human blood over a period of 2 hours, after which responses remained essentially stable. Whole blood patient glucose determinations yielded a correlation coefficient of $r^{2}=0.99$ compared to standard hospital analyses.

Keywords: cathodic electropolymerisation, composite membrane, amperometric enzyme electrode, polydivinylbenzene, blood. 


\section{Introduction}

Glucose continues to be a major target analyte for many sensors, with the focus of much of this research being driven by the growing incidence of diabetes, at the time of writing 170 million sufferers have been diagnosed world-wide [1] and this number is expected to reach 300 million by 2045 [2].

When measuring whole blood samples, two problems must be overcome: Firstly interferents that may be present in the blood (such as ascorbate) must be prevented from reaching the electrode since they can be oxidised and give rise to erroneous readings. Secondly physiological fluids, especially blood, also have a tendency to deposit materials such as proteins, usually irreversibly, onto solid surfaces. This biofouling process can diminish the response of sensors - and in some cases can passivate them completely. The solution is often to employ two separate functional membranes [3]; an outer membrane to act as a substrate diffusion limiting barrier and offer favourable biocompatibility [4] along with an underlying (inner) membrane that is designed to be permselective [5] and which is typically positioned immediately over the working electrode to screen out interferents. Unfortunately both membranes act as additional diffusional barriers through which the enzyme substrates and/or $\mathrm{H}_{2} \mathrm{O}_{2}$ must traverse, so hindering their passage to the enzyme or working electrode and thus increasing the overall response time.

This has lead to research towards the development of single multi-functional ultra thin film composite membranes [6,7]. Membranes of this type have been shown to be capable of serving as both highly substrate diffusion limiting barriers towards enzyme substrates, whilst also effectively screening anionic interferents from the working electrode via a charge exclusion principle [8]. The usual method for formation of composite membranes of this type is to deposit a homogeneous film 
(ideally $100 \mathrm{~nm}$ thickness) of a biocompatible polymer onto a highly porous support membrane, which offers mechanical support to the structure. Amperometric glucose oxidase laminate enzyme electrodes employing such biocompatible composite membranes have been found to display shortened response times [9].

It should be noted in this context that the separational performance of a homogeneous polymer film membrane relies on its chemical properties and not on its thickness per se, whereas the rate at which the separation can occur is dependent on the thickness of the membrane. It therefore follows that the thinner a membrane is, the better will be its performance also. On the other hand it should be appreciated that if a membrane is made too thin, it will become excessively fragile and thus be rendered useless. A membrane of substantially reduced thickness which could offer the required separational performance, whilst still possessing the mechanical strength of a conventional contemporary membrane, would therefore clearly be highly advantageous.

Electrodeposition affords the possibility of accurate control of the location and thickness of a deposited polymer film. The active species e.g. an enzyme can be incorporated within the polymeric film [10].

A second approach is to combine an active layer such as a chemically crosslinked film of glucose oxidase - which is either directly deposited onto or laminated to a supported electro-deposited membrane on an electrode. Once deposited, this active layer is then covered with the permselective composite membrane which both screens out interferents and offers favourable biocompatibility. Previous work within out group has utilised highly porous commercial alumina membranes as supports for ultra-thin polydivinylbenzene/ethylvinylbenzene (DVB/EVB) or polyacrylonitrile films [11,12]. However the alumina support 
membranes were somewhat inflexible and brittle. The next aim of this work was focussed towards developing this sensor further by increasing its mechanical flexibility and so increasing the possible scope of application within sensors for practical applications.

This paper describes the development and use of an ultra-thin polydivinylbenzene/ethylvinylbenzene (DVB/EVB) film composite membrane, with an effective diffusional barrier thickness of approximately $50 \mathrm{~nm}$, for the optimisation of a glucose biosensor for whole blood glucose determinations. The performance of a variety of composite membranes as substrate diffusion limiting barrier were determined. The biocompatibility as well as the ability to screen anionic interferent solutes within patient blood samples were also investigated. A model glucose oxidase enzyme electrode was also fabricated for the determination of glucose levels in patient whole blood samples. The results are compared to standard hospital analyses for the same patient samples. The advantages of this system over many of the other glucose sensors reported are the control of membrane thickness conferred by electrochemical deposition and the flexibility of the resultant glucose sensor.

\section{Experimental}

\subsection{Reagents and Membranes}

N'N-dimethylformamide, tetrabutylammonium perchlorate (electrochemical grade) and divinylbenzene (70-85\% DVB, $15-30 \% \quad 3-$ and 4- EVB, containing $0.1 \%$ polymerisation inhibitor: 4-tert-butylcatechol) were all purchased from Fluka Chemicals (Dorset, England). 
D-glucose, disodium hydrogen phosphate $\left(\mathrm{Na}_{2} \mathrm{HPO}_{4}\right)$, sodium dihydrogen phosphate $\left(\mathrm{NaH}_{2} \mathrm{PO}_{4}\right)$ and sodium chloride $(\mathrm{NaCl})$ were all obtained from $\mathrm{BDH}$ (Poole, Dorset). Glucose oxidase from Asperigillus niger (75\% protein 150,000 units/g solid), bovine serum albumin (fraction V) and glutaraldehyde (grade II, 25\% aqueous solution) were all purchased from the Sigma Chemical Company (Poole Dorset, UK).

Aluminium oxide Chromatography Grade was obtained from F.S.A. Laboratory Supplies (Loughborough, UK). Granular calcium hydride was purchased from Fisher Scientific (Leicestershire, UK).

Electrolube ${ }^{\circledR}$ silver conductive paint (Electrolube ${ }^{\circledR}$ Ltd, Berkshire) was purchased from Maplins Electronics, Manchester, UK and used for the fabrication of Au counter electrodes. ElectroDag® silver paint was purchased from Agar Scientific Ltd, for electron microscopy sample preparations.

Cyclopore $^{\mathrm{TM}} 0.2 \mu \mathrm{m}$ and $0.4 \mu \mathrm{m}$ polyester membranes, $0.2 \mu \mathrm{m}$ PTFE membranes, $0.2 \mu \mathrm{m}$ nylon membranes and $0.45 \mu \mathrm{m}$ polypropylene membranes were purchased from Whatman International, Maidstone, UK. $0.015 \mu \mathrm{m}$ pore diameter polycarbonate membranes (Poretics Corporation, Livermore, USA) were used as the lower membranes within enzyme/membrane laminates [9].

\subsection{Buffers and Solutions}

A phosphate buffer ( $\mathrm{pH} 7.4$ ) comprising $5.28 \times 10^{-2} \mathrm{M} \mathrm{Na}_{2} \mathrm{HPO}_{4}, 1.3 \times 10^{-3} \mathrm{M}$ $\mathrm{NaH}_{2} \mathrm{PO}_{4}$ and $5.1 \times 10^{-3} \mathrm{M} \mathrm{NaCl}$ in deionised water was used for the preparation of all solutions for diffusion chamber mass transport investigations and glucose calibration measurements. 


\subsection{Preparation of Conductive faced Membrane Electrodes and Counter Electrodes}

In order to electropolymerise the DVB/EVB monomer onto the host membranes, the uppermost surface was first rendered electrically conductive by Au sputter coating the membranes with an Edwards S150 B sputter coater in a similar manner as used for electron microscopy sample preparation [8]. The gold-coated membranes were then attached to multicore wires using fluxless solder and were then used as working electrode assemblies for the electrochemical preparation of thin polymer film composite membranes.

Gold counter electrodes were constructed by coating glass slides in a similar manner and attaching multicore wires via the application of 'Electrolube' silver conductive paint. A further coating of epoxy resin served to insulate the wire/electrode joint, whilst also enhancing the mechanical strength of the electrode.

\subsection{Removal of Polymerisation Inhibitor present in as-received Divinylbenzene}

Prior to the polymerisation of the divinylbenzene, the polymerisation inhibitor (4-tert butylcatechol) was removed from the as-received monomer as previously described [11]. It must be noted, however, that the monomer preparation consisted of a mixture of $70-85 \%$ divinylbenzene plus $0.1 \%$ polymerisation inhibitor with the rest of solution constituting 3- and 4-ethylvinylbenzene. Since no attempt was made to separate the DVB from the EVB, the resulting polymer films obtained are copolymers of the two styrenic monomers.

The as-received DVB/EVB was extracted 10 times with $5 \%$ aqueous sodium hydroxide to remove the 4-tert-butylcatechol. The extract produced was washed six 
times with high purity de-ionised water and then passed through a column of neutral activated aluminium oxide onto granular calcium hydride. The inhibitor free divinylbenzene was then stored at $4^{\circ} \mathrm{C}$ over calcium hydride prior to use.

\subsection{Enzyme Laminate Fabrication}

The enzyme laminate employed in the resulting enzyme electrode was produced by first forming a GOD/BSA solution comprising glucose oxidase (2560 units/ml) and bovine serum albumin $\left(0.1 \mathrm{mg} \mathrm{ml}^{-1}\right)$ dissolved in buffer. $3 \mu 1$ glutaraldehyde $(5 \% \mathrm{v} / \mathrm{v}$ in buffer) was then added to $6 \mu \mathrm{l}$ GOD/BSA solution and mixed rapidly. The enzyme/glutaraldehyde mixture was then placed on a $1 \mathrm{~cm}^{2}$ portion of $(0.015 \mu \mathrm{m}$ pore $)$ polycarbonate membrane and a second $1 \mathrm{~cm}^{2}$ portion of the polydivinylbenzene composite membrane (polymer coating face up) placed on top. Using glass slides, the crosslinked enzyme laminate was then compressed under gentle finger pressure for approximately 5 minutes.

\subsection{Enzyme electrode glass cell for whole blood glucose determinations}

A bespoke glass electrochemical cell was utilised as before [11], Figure 1, for enzyme electrode blood glucose determinations. This glass cell consisted of two halves clamped together. The base was constructed with a flat circular surface, at the centre of which was a platinum working electrode of $2 \mathrm{~mm}$ diameter. The polymer coated composite membrane laminate was positioned over the platinum electrode of the cell (polarised at $+650 \mathrm{mV}$ vs. $\mathrm{Ag} / \mathrm{AgCl}$ ) and sealed in place with an 'O-ring' to follow the detection of $\mathrm{H}_{2} \mathrm{O}_{2}$. The upper half of the cell acted as a chamber into which solutions and reference and counter electrodes were introduced. A circular hole in the base of 
this served to expose the underlying working electrode to the solution. A further circular indentation was made in the underside surface of the solution well to house the O-ring. A silver electrode was placed vertically into the main chamber of the cell to act as a combined counter and reference electrode. The enzyme laminate was positioned over the Pt working electrode with the polymer-coated surface positioned uppermost.

\subsection{Investigation into Solute Permeability Coefficients}

The mass transport, and therefore permeability coefficients, of both oxygen and glucose across a range of ultra-thin polymer composite membranes were determined using conventional diffusion chamber apparatus as previously described [5]. A Rank Oxygen Electrode assembly (as previously described [5]) was used for the determination of oxygen levels within solution, whilst a glucose enzyme electrode (again as previously described [5]), was fabricated for the determination of glucose levels.

\subsection{Scanning Electron Microscopy}

Bare polymer membranes and a range of polymer coated composite membranes were studied using a Phillips XL30 FEG Scanning Electron Microscope. Samples were mounted onto aluminium microscopy stages by applying a small amount of silver conductive 'ElectroDag' $(415 \mathrm{M})$ paint around the sample perimeter.

\section{Results}

\subsection{Selection of membrane.}


The membranes were tested to determine their permeability to glucose. It is intended that the polymer film acts as the substrate diffusion limiting factor in the assembly, not the membrane itself, and so the ideal membrane chosen for this study would act as a highly permeable, microporous mechanical support for the polymer film. It was therefore intended that the membrane found to posses the greatest permeability to glucose solution should be chosen.

The polypropylene and PTFE membranes were so hydrophobic that the glucose solution could not penetrate the membrane and zero permeability was observed. The highest permeability was observed for the $0.4 \mu \mathrm{m}$ polyester membrane (12.5 $\left.\times 10^{-7} \mathrm{~cm}^{2} \mathrm{~s}^{-1}\right)$ compared with $3.8 \times 10^{-7} \mathrm{~cm}^{2} \mathrm{~s}^{-1}$ for the $0.2 \mu \mathrm{m}$ polyester membrane, $2.5 \times 10^{-7} \mathrm{~cm}^{2} \mathrm{~s}^{-1}$ for the nylon membrane and $9.7 \times 10^{-7} \mathrm{~cm}^{2} \mathrm{~s}^{-1}$ for the alumina membranes studied previously [11]. Therefore the $0.4 \mu \mathrm{m}$ polyester membrane was selected as the substrate for sensor optimisation.

\subsection{The Electrodeposition of polyDVB/EVB onto Polyester Host Support}

\section{Membranes}

Earlier work with polyDVB/EVB-coated alumina membranes [11] demonstrated the most dramatic changes in mass transport properties occur following the deposition of polymer within the first few potential cycles, with the relative difference between $P$ values for membranes coated via 5 or 50 potential cycles being small. In this work membranes (with a nominal pore diameter of $0.4 \mu \mathrm{m}$ ) were for this reason coated using 1, 2, 3, 5 and 10 potential cycles. 
The cyclic voltammograms obtained for the electrodeposition of polyDVB/EVB onto polyester are shown in Figure 2 and are dissimilar to the voltammograms produced via deposition on alumina [11].

\subsection{Investigation of Surface Topography using the Scanning Electron Microscope}

Scanning electron microscopy images show the polyester membrane surface to be covered by a coating of polymer after 2 potential sweeps (Figure $3 b$ ), in comparison to the bare membrane (Figure 3a). The underlying trans-membranous pores are still visible in the structure, since the polymer surface follows the contours of the membrane, producing a polymer surface that appears 'pitted'. This may be explained by taking into consideration that the formation of polymer also occurs within the trans-membranous pores. These pores create channels in which diffusion of soluble polymer is limited, resulting in an increased concentration of soluble polymer molecules forming and becoming contained within each pore. These areas within the pores that possess a high concentration of soluble polymer will reach the critical gel-point of polymerisation earlier than in areas such as the membrane surface, where diffusion away from the electrode is possible. By reaching the critical gel-point at an earlier stage, the polymer layer formed within each pore will be thinner than that formed at the surface, where the potential window for soluble polymer formation is greater. The 'pitted' appearance of the polymer film will not, however, affect the permeability properties of the membrane, since the passage of substrates through the membrane occurs only through these pores, which, if blocked, will still act as effective substrate diffusion limiting barriers. Figure $3 \mathrm{c}$ shows the surface of a polyester membrane coated with polyDVB/EVB via 10 potential scans, in which the pores are clearly blocked. 


\subsection{Investigation into film thickness via Charge Integration and SEM Techniques}

The cumulative thickness of polymer film deposited was estimated by integrating the charge underneath each polymerisation curve [11]. Assuming via molecular modelling that the cross-sectional dimensions of a polyDVB/EVB monomer unit are $\sim 8.06 \AA$ and $\sim 5.28 \AA$ respectively and that the monolayer depth is $\sim 1.5 \AA$ - the polymer film thickness may be predicted to be less than $50 \mathrm{~nm}$.

A scanning electron micrograph of the cross section of a polyester membrane coated via 10 sweeps shows the polymer film thickness to be approximately $50 \mathrm{~nm}$, indicating that an ultra-thin-polymer composite membrane has been fabricated (Figure 3d).

\subsection{Enzyme Immobilisation}

The prototype sensor described in this chapter was designed to electrochemically quantify the levels of glucose present in whole blood, and so the enzyme glucose oxidase was immobilised at the working electrode surface via crosslinking with bovine serum albumin. The immobilised enzyme was then entrapped between a polycarbonate membrane and an upper polyDVB/EVB-coated polyester membrane.

In the same way as the sensors designed previously [11], the prototype sensor relies on the catalytic oxidation of glucose by the enzyme glucose oxidase, a reaction that requires both glucose and oxygen. The mass transport properties of both substrates were therefore investigated in order to identify the most suitable membrane for the application. 


\section{6. $\quad$ The Determination of Permeability Coefficients}

The first few potential sweeps are responsible for the blocking of the membrane pores, after which successive potential cycles contribute to the cumulative thickness of solid polymer formed. It must be remembered here that although the surface of a membrane coated via 2 cycles may still appear to possess some unblocked pores (Figure 3b), this may not be the case. It is possible that thin films of polymer may have formed within these pores that are not yet thick enough to be visible via electron microscopy at the membrane surface.

Glucose $P$ values decrease steadily with successive potential cycles, reinforcing the theory that polymer is being progressively deposited at the membrane surface. The glucose $P$ values depicted in Figure 4a show a sudden drop occurring following the application of the first potential cycle, however this may be attributed to the blocking of membrane pores, so that glucose must partition through the polymer film itself, rather than within aqueous solution. Not all membrane pores may be completely blocked at this point, however, since the $P$ values for oxygen indicate that it is after the second potential cycle that the mass transport of oxygen molecules through the membrane is most greatly hindered.

The oxygen $P$ values also decrease (Figure $4 \mathrm{~b}$ ) with each successive polymer coating applied as the film coats the underlying membrane with increasing layers of polymer. The greatest lowering of the permeability coefficients for the mass transport of oxygen through the membrane occurs following the initial first and second potential cycles. After this point the $P$ values decrease at a lowered rate with each successive potential sweep.

This difference in $P$ values can be explained by the larger glucose molecule meeting increased resistance to the diminishing pore size and thickening polymer film 
after the first potential cycle. The smaller oxygen molecule may still be able to diffuse through any small pinhole areas of the pores that remain unblocked, whereas the glucose must partition through the polymer film itself. Following the application of the second polymerisation potential cycle, both substrates must partition across the polymer film in order to traverse the membrane.

This mass transport behaviour would suggest that, although not visible at the surface, the vast majority of the membrane pores have some amount of polymer formed within or across them after 2 potential cycles.

After approximately 10 potential sweeps, a small number of the membranes studied began to show some deterioration, with the surface of the polymer-coated gold layer exhibiting some cracking (Figure 5). This, in turn, affected the reproducibility of the oxygen and glucose $P$ values for these membranes.

The glucose/oxygen $P$ ratios for the range of polyDVB/EVB-coated polyester membranes are shown in Figure 4c. It has been shown that the passage of glucose through the membrane is most greatly affected after the application of the first potential cycle, and this is reflected in the initial large drop in glucose/oxygen $P$ ratio. The main mechanism of mass transport of glucose through this membrane involves partitioning through the polymer film. It is possible however, that at this point the smaller oxygen molecule may still be traversing the membrane through small holes in the pores that remain unblocked. The relative increased resistance offered to the oxygen molecules after the second potential cycle therefore causes a slight increase in the glucose/oxygen $P$ ratio, as partitioning becomes the primary method of mass transport for both enzyme substrates.

Following the third potential cycle, all membrane pores become blocked by polymer and so with subsequent polymer layers deposited, the glucose/oxygen $P$ ratio 
continues to decrease steadily. After 10 potential cycles have been performed the glucose/oxygen $P$ values increase. This is due to some membranes showing deterioration, as depicted in Figure 5.

\subsection{Calibration and Comparison of a Range of Glucose Sensors Incorporating} Flexible Thin-Film PolyDVB/EVB Composite Membranes

A range of glucose oxidase enzyme electrodes were constructed, with each electrode employing a flexible composite membrane produced via 1, 2, 3, 5 and 10 potential sweeps. Calibration curves were obtained over a concentration range of 0mM to $30 \mathrm{mM}$ glucose in phosphate buffer (Figure 6). All membranes served as effective limiting barriers to the diffusion of both the enzyme substrates and for each successive polymer coating deposited the amperometric responses recorded became progressively linearised. Response times for all sensors were found to be $<1$ minute. Sensors with as few as 2 layers showed good response and linearity but tended towards a plateau above $20 \mathrm{mM}$ glucose; thicker films such as 5 layers displayed lower responses but enhanced linearity beyond $30 \mathrm{mM}$ glucose.

\subsection{Exclusion of Electroactive Interferents Present in Human Whole Blood}

As described previously [11] the highly substrate diffusion limiting composite membranes based on alumina membranes permitted the effective exclusion of anionic interferents due to a charge exclusion principle. The composite membranes produced in this work consisted of polyDVB/EVB coated onto polyester support membranes, which functioned as effective substrate diffusion limiting barriers. A flexible ultra-thin polyDVB/EVB composite membrane coated via 5 potential cycles was 
employed as the upper covering membrane in a whole blood glucose sensor, since this membrane displayed the lowest glucose/oxygen $P$ ratio, along with good polymer coverage and increased linearisation of response. The enzyme electrode was then evaluated for its ability to screen anionic interferents in this way.

Ascorbate is normally used as the most significant compound with glucose sensors in tests of interference. The sensor was exposed to glucose both in the absence and presence of $1 \mathrm{mM}$ ascorbate and it was found that the enzyme electrode response was not significantly affected by the presence of ascorbate until $15 \mathrm{mM}$ glucose was added. The response was found to increase by $5 \mathrm{nA}$ at a concentration of $15 \mathrm{mM}$ glucose and $7 \mathrm{nA}$ after $30 \mathrm{mM}$ glucose was added, therefore demonstrating screening of ascorbate under conditions of lower glucose concentration in a similar manner to previous findings within our laboratory $[6,8,11]$. In this context we have previously shown that under conditions of low glucose flux, the anionic enzyme/albumin matrix prevented the passage of anionic solutes to the underlying electrode [6]. It appears that interferent contributions (due to a fixed concentration) only become significant when the enzyme layer experiences a higher glucose flux. Here, it is probable that despite ambient solution buffering, the enzyme catalysed production of gluconolactone (and therefore gluconic acid) will lower the local enzyme layer $\mathrm{pH}$, and the protective barrier action of the enzyme layer will be diminished [6]. The effects of ascorbate under conditions of high glucose flux are lower for the membranes described here than for the alumina based membranes [11], perhaps indicating a better quality film and more effective exclusion of ascorbate. 


\subsection{Assessment of Composite Membrane Performance within a Working Glucose}

Sensor for Human Whole Blood Determinations

In the same way as described earlier [11], a GOD enzyme electrode possessing a polyDVB/EVB-coated (5 cycles) upper covering membrane was used for the construction of a working sensor for whole blood glucose determinations. The enzyme electrode was exposed to whole blood for 2 hours prior to patient sample determinations for glucose. Responses to $5 \mathrm{mM}$ glucose buffer samples indicated a loss of approximately $10 \%$ during this time - after which time the response of the sensor remained almost stable. Previous results have indicated that enzyme electrodes possessing stable baselines facilitate the analysis of multiple blood samples. This loss of enzyme electrode response observed during the first two hours of exposure to blood is caused by initial biofouling effects and need not cause any further problems with analyses. The sensors described earlier [11] experienced a greater loss of response $(32 \%)$ following its exposure to blood. This may be due to the polyester membrane offering enhanced haemo/biocompatibility over the alumina membrane.

No further loss of response was observed following this period and so it may therefore be treated as a pre-conditioning step. Enzyme electrodes were therefore pre-exposed to blood and re-calibrated prior to the determination of blood glucose samples. Patient blood samples were supplied by a local hospital biochemistry laboratory and stored in fluoride-oxalate containers to prevent glucose metabolism via the blood cells prior to analyses. Blood glucose analyses via the enzyme electrode are compared in Figure 7 to standard hospital biochemistry laboratory analyses for the same samples. The blood glucose analyses via the enzyme electrode showed a very close correlation of $\mathrm{r}^{2}=0.99$ when compared to the hospital laboratory analyses. This 
is superior to the results for alumina membranes [11] which gave a correlation of $r^{2}=0.97$.

\subsection{Investigation of Membrane Surface Biofouling Effects}

Polyester is a highly biocompatible material and is, for example, used to surgically replace artificial blood vessels $[12,13]$. Scanning electron microscopy images of bare polyester membranes that have been exposed to blood for a period of 2 hours showed no obvious biofouling (Figure 8a). The polyDVB/EVB-coated alumina membranes have already been shown to offer enhanced haemo/biocompatibility [11] and polymer-coated polyester membranes again offer this characteristic. Figure $8 \mathrm{~b}$ shows a scanning electron microscopy image of a polyDVB/EVB-coated polyester membrane following exposure to whole human blood for 2 hours.

\section{Conclusions}

Flexible ultra-thin-polyDVB/EVB composite membranes have been electrochemically fabricated and successfully employed as the outer covering barrier in a model glucose sensor for whole blood glucose determinations, demonstrating their applicability for sensor systems.

Scanning the working electrode potential between $0 \mathrm{~V}$ and $-4 \mathrm{~V}$ (vs. Ag) via $1,2,3,5$ and 10 cycles produced a range of composite membranes. As the pores of the underlying polyester membranes became increasingly blocked with polymer, the glucose $/ \mathrm{O}_{2} P$ ratios decreased, as both solutes begin to partition through the polymer film to reach the enzyme matrix.

The enzyme electrode was fabricated using a composite membrane produced via 5 potential cycles and calibration curves obtained for known quantities of glucose. The composite membrane was found to serve both as an excellent substrate diffusion 
limiting barrier and the linearity of sensor responses was found to increase with increasing polymer coatings. The use of such composite membranes proved to be an effective means for screening against electroactive interferents via a charge exclusion mechanism. The underlying polyester membrane proved to be a suitably biocompatible support for the polymer film, which also displayed favourable biocompatibility, thereby helping minimise surface biofouling effects. Compared with our previous work on alumina membranes [11], the polyester membrane sensors within this work displayed superior physical properties (flexible, non-brittle) and better correlation between sensor response and standard hospital analyses for glucose in whole blood samples. Future work could involve use of other electrodeposited materials to optimise performance and exclusion of interferents.

\section{Acknowledgements}

The authors would like to thank the EPSRC for a PhD studentship for K. Bridge, the BBSRC for funding for F. Davis as part of the Centre for Bioarray innovation within the post-genomic consortium and the Nuffield Foundation for further financial support. We would also like to thank Mr. R. Hinchcliffe of the Manchester Royal Infirmary for supplying blood samples for comparative testing. 


\section{References}

[1] World Health Organisation, www.who.org

[2] J. D. Newman, L. J. Tigwell, A. P. F. Turner, P. J. Warner, 2004, Biosensors: A Clearer View, Biosensors 2004 - The 8th World Congress on Biosensors. Elsevier.

[3] W. R. Veith (Ed). Analysis and Design, 1998.

[4] W. H. Mullen, F. H. Keedy, S. J. Churchouse, P. M. Vadgama, Anal. Chim. Acta, 1986, $183,59$.

[5] I. M. Christie, P. H. Treloar, P. M. Vadgama, Anal. Chim. Acta, 1992, 269, 65.

[6] S. P. J. Higson, P. M. Vadgama, Anal. Chim. Acta, 1995, 300, 85.

[7] B. Ballarin, C. Brumlik, D. R. Lawson, W. Liang, L. S. Van Dyke, C. R. Martin, Anal. Chem, 1992, 64, 2647.

[8] S. Myler, S. Eaton, S. P. J Higson, Anal. Chim. Acta, 1997, 357, 55.

[9] S.P.J. Higson, P.M. Vadgama, Anal. Chim. Acta, 1995, 300, 77.

[10] S. Cosnier, Anal. Bioanal. Chem, 2003, 377, 507.

[11] K. Bridgẹ, F. Davis, S. D. Collyer, S. P. J. Higson, Electroanalysis, 2006, 18, 95.

[12] S. Wakabayashi, T. Hashimukai, T. Katakura Y. Takano, Composites Part A: Applied Science and Manufacturing, 28, Issue 6, 1997, 611-612.

[13] P. M. Portner, P. G. M. Jansen, P.E. Oyer, D. R. Wheeldon, D.R. \& N. Ramasamy, The Annals of Thoracic Surgery, 2001, 71, 205. 


\section{LEGENDS FOR FIGURES.}

Figure 1. Schematic of Electrochemical Cell.

Figure 2. Cyclic voltammograms for electrochemical deposition of polyDVB/EVB onto host support membrane (at a scan rate of $50 \mathrm{mV} \mathrm{s}^{-1}$ ).

Figure 3. SEM image of the surface of a polyester membrane (a); surface view of the membrane as supplied, (b); surface view of the membrane partially coated with polyDVB/EVB following 2 potential cycles, (c); surface view of the membrane coated with polyDVB/EVB following 10 potential cycles and (d) a cross-section of a membrane coated with poly DVB/EVB following 10 potential cycles.

Figure 4. Permeability coefficients across polyDVB/EVB coated thin polymer film composite membranes for (a); glucose, (b); oxygen and (c); the glucose/oxygen permeability ratios across polyDVB/EVB coated membranes.

Figure 5. SEM image of the surface of a polyester membrane coated with polyDVB/EVB following 10 potential cycles showing presence of some deterioration in polymer film quality.

Figure 6. Glucose enzyme electrode calibrations employing membranes coated via 0

$(\bullet), 1(\mathbf{\square}), 2(\mathbf{A}), 3(\times), 5(ж)$ and $10(\bullet)$ potential cycles. 
Figure 7. Whole blood glucose determinations for patient samples via enzyme electrodes employing 5 potential cycles in comparison to standard hospital results.

Figure 8. SEM images depicting the effects of blood biofouling on (a); unmodified polyester membrane surface (b); polyDVB/EVB coated polyester membrane surface. 

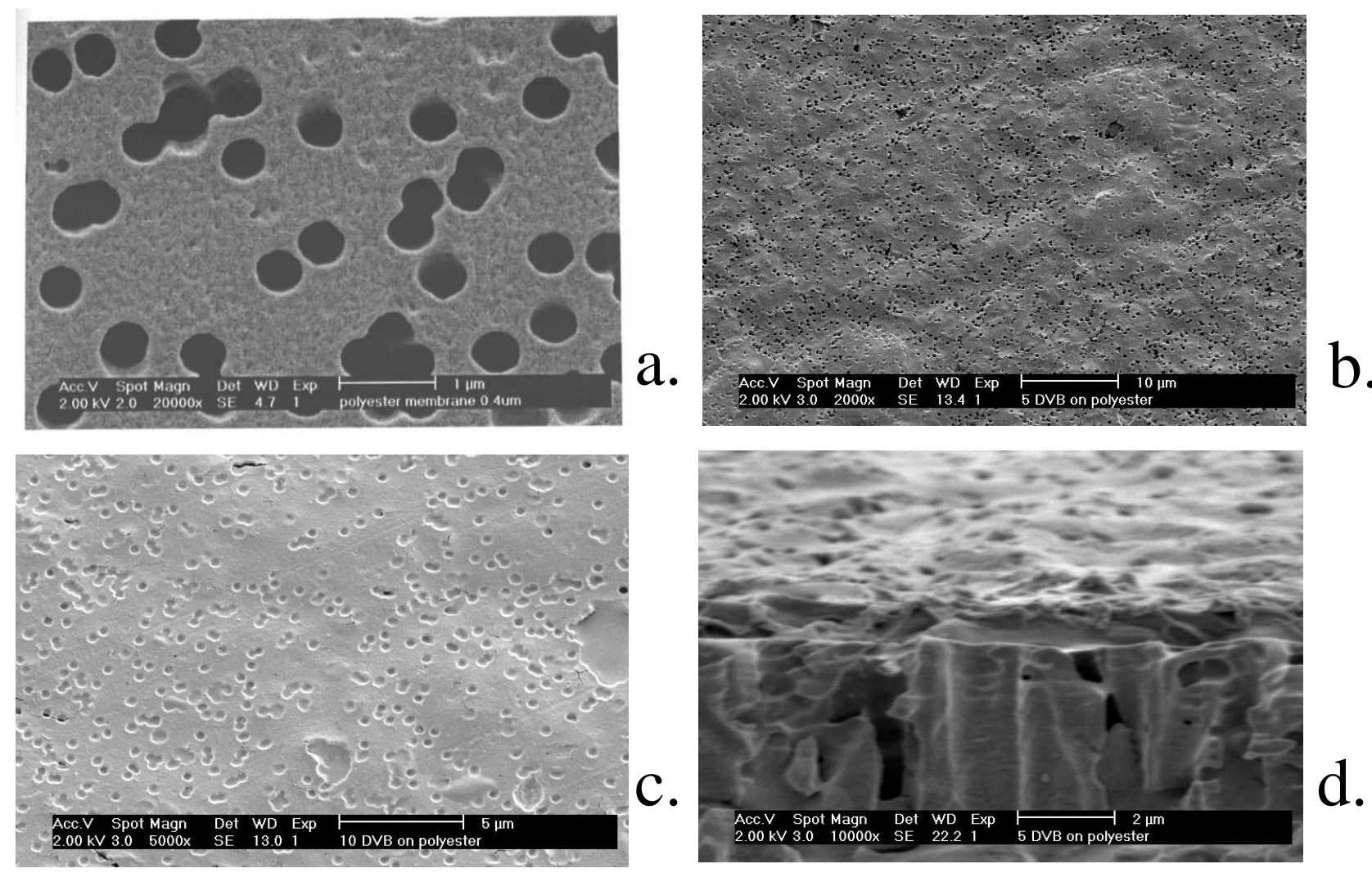


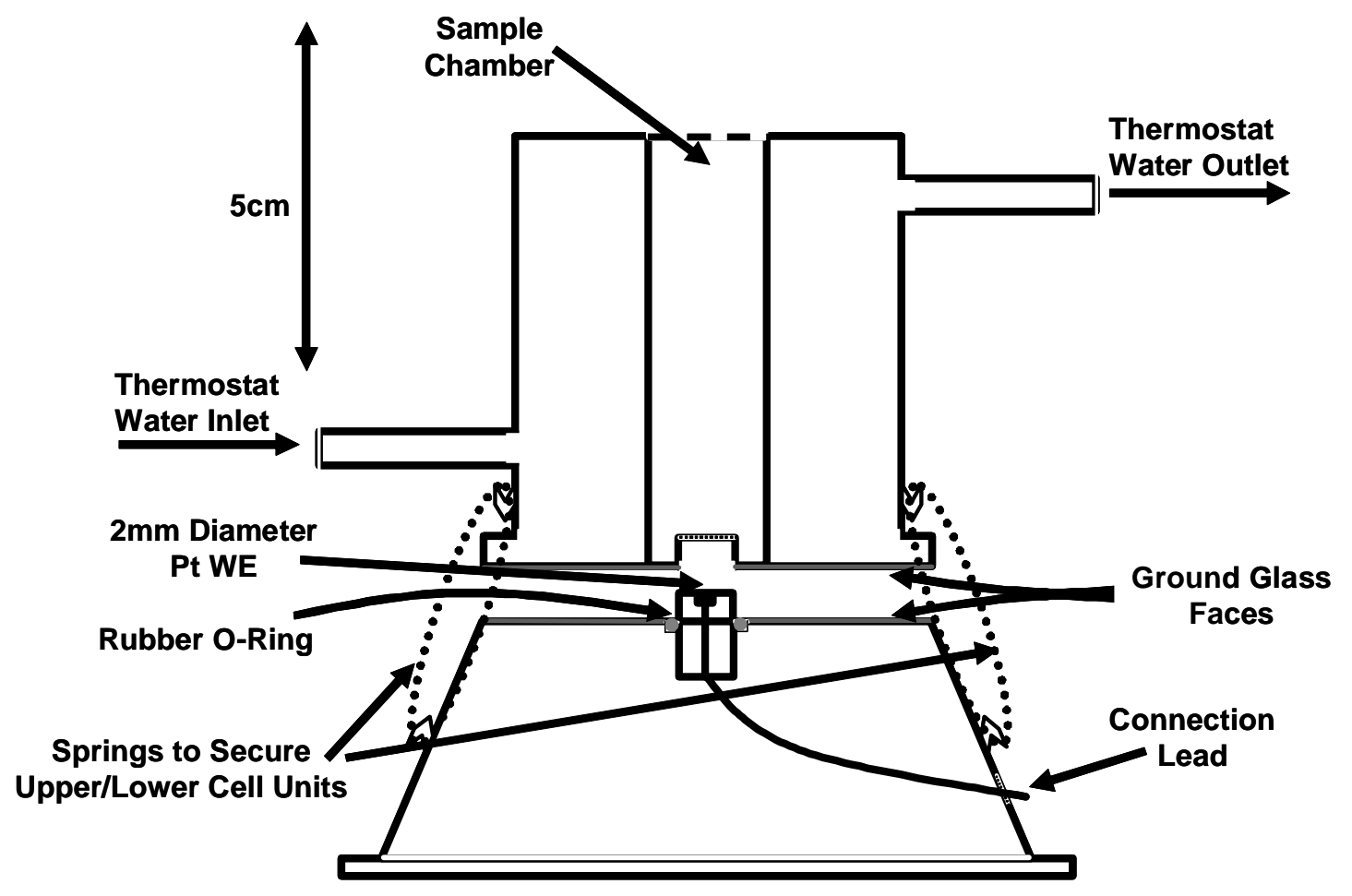




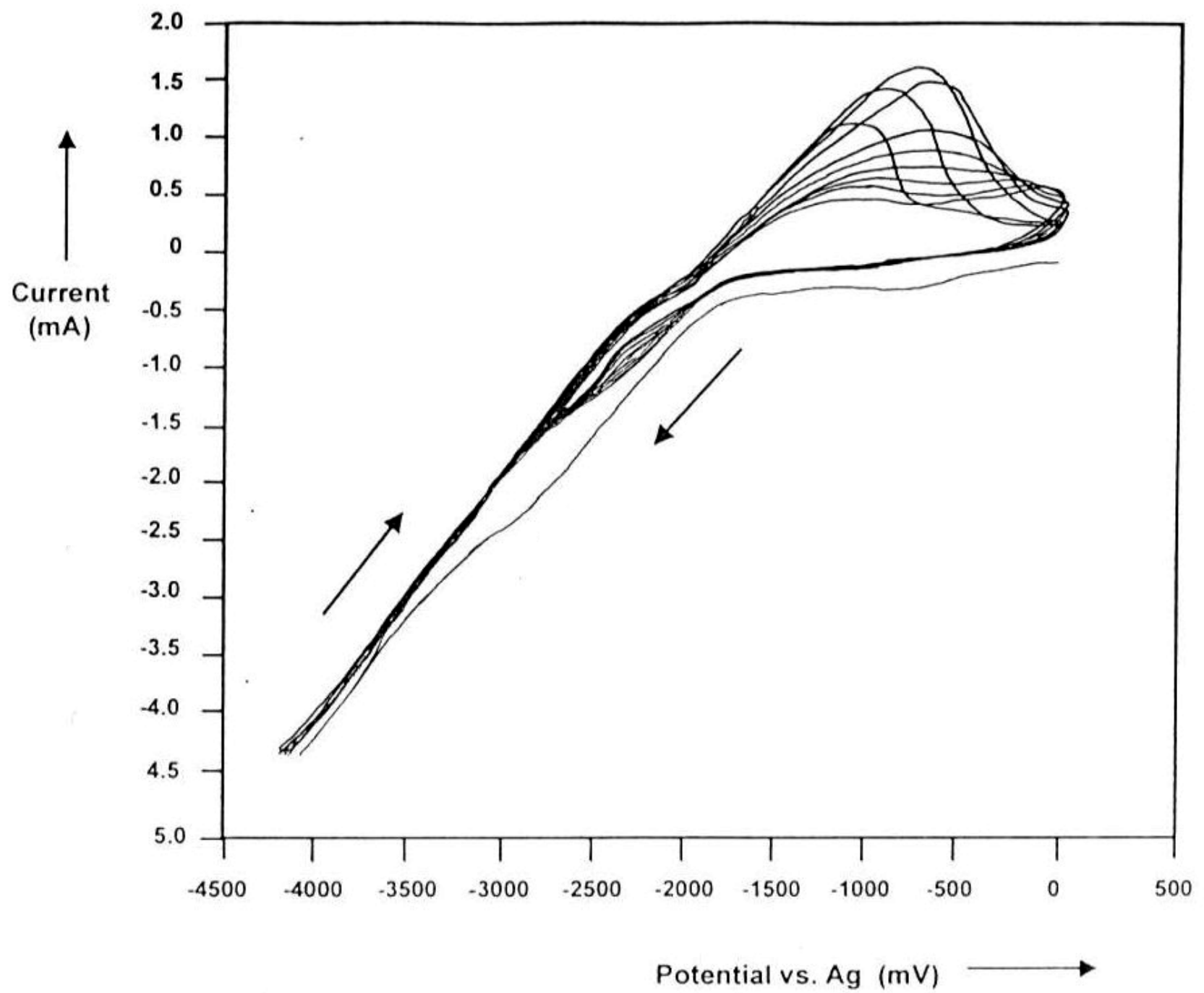




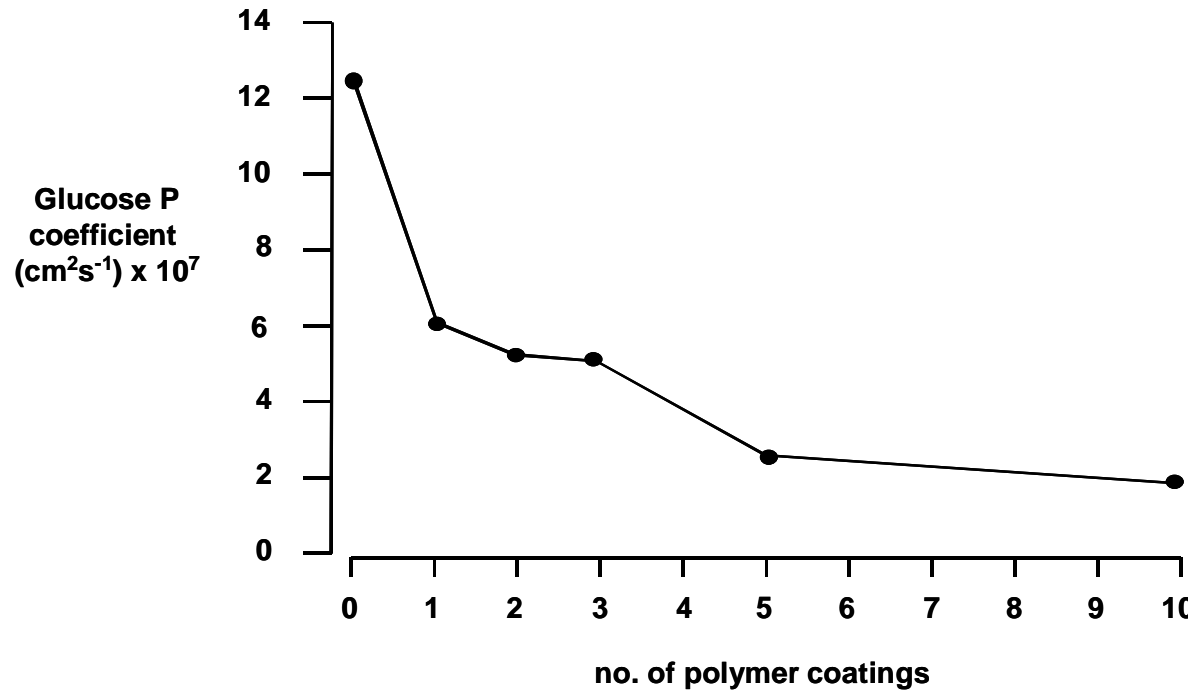

a.

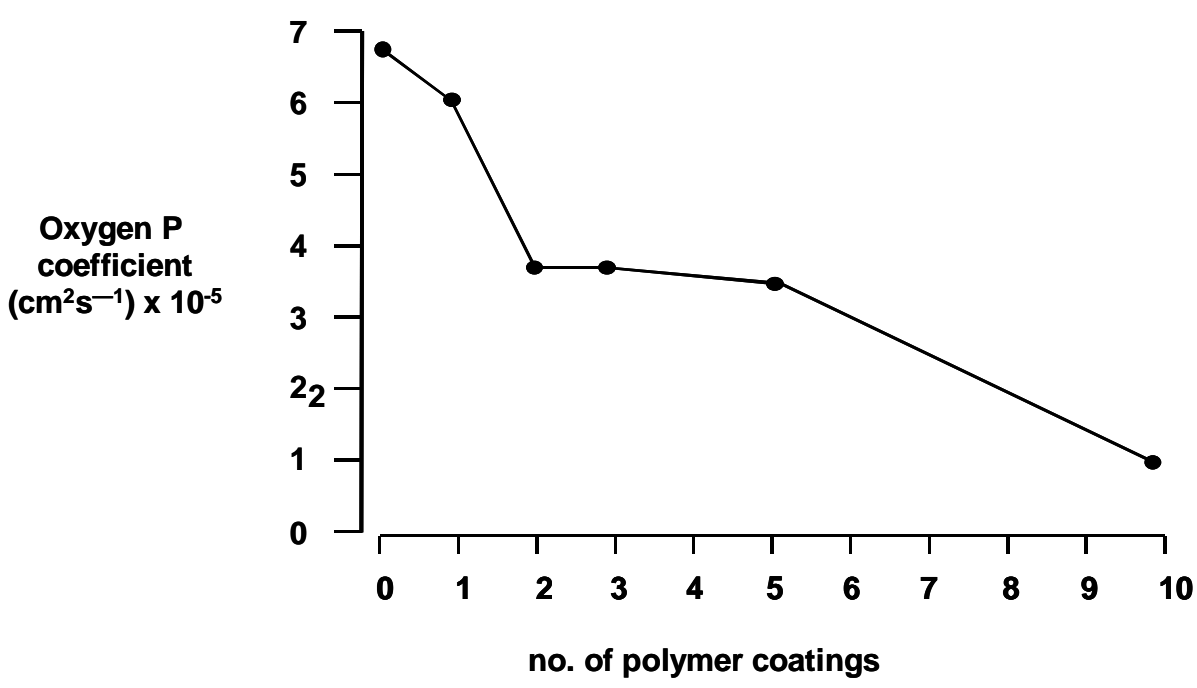

b.

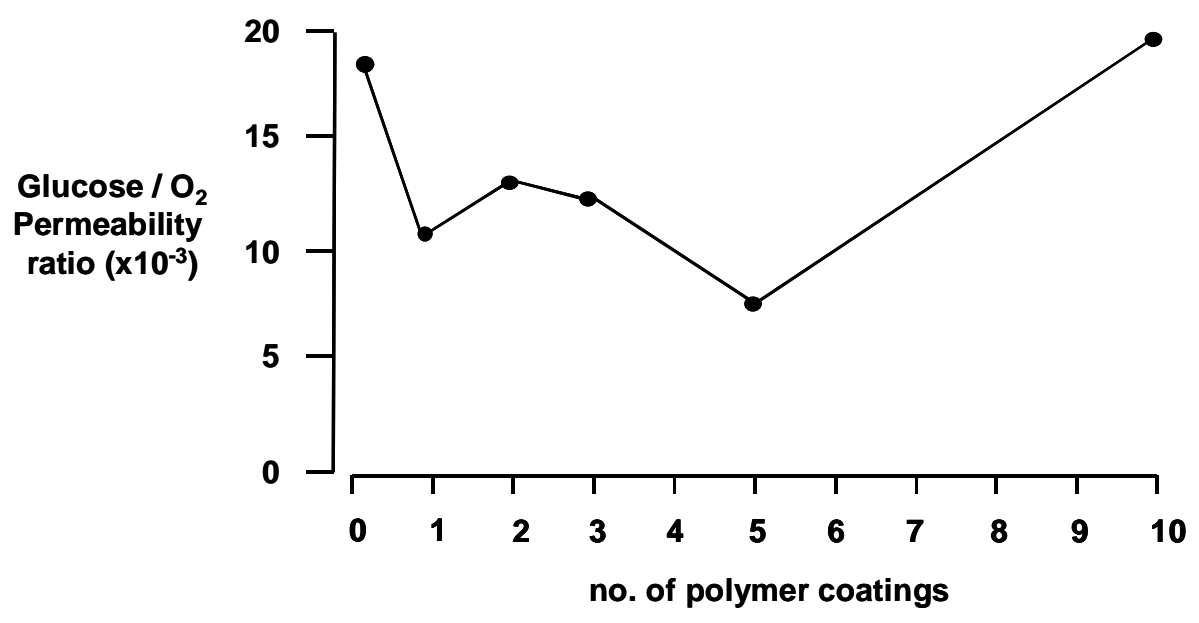

c. 


\section{Cracks on surface}

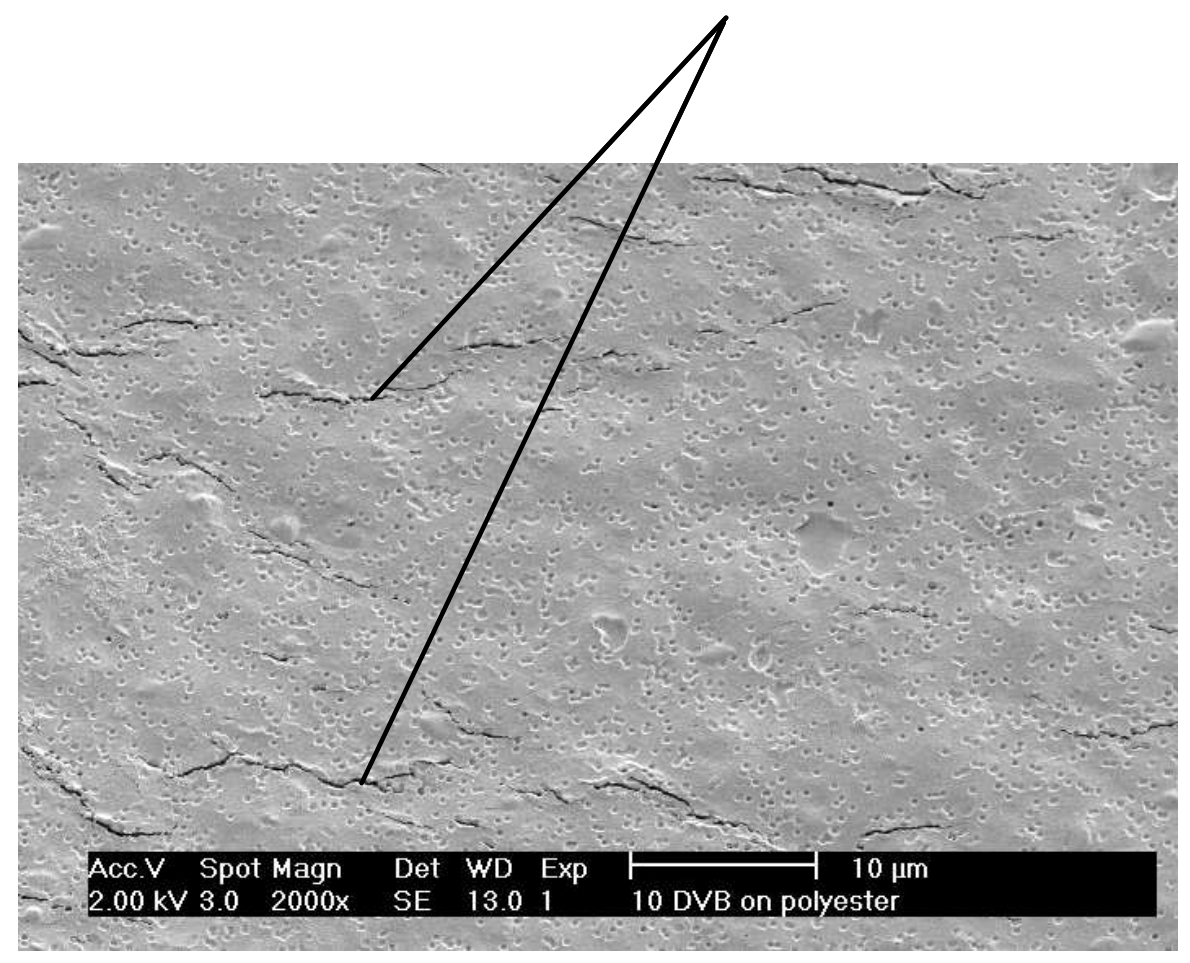




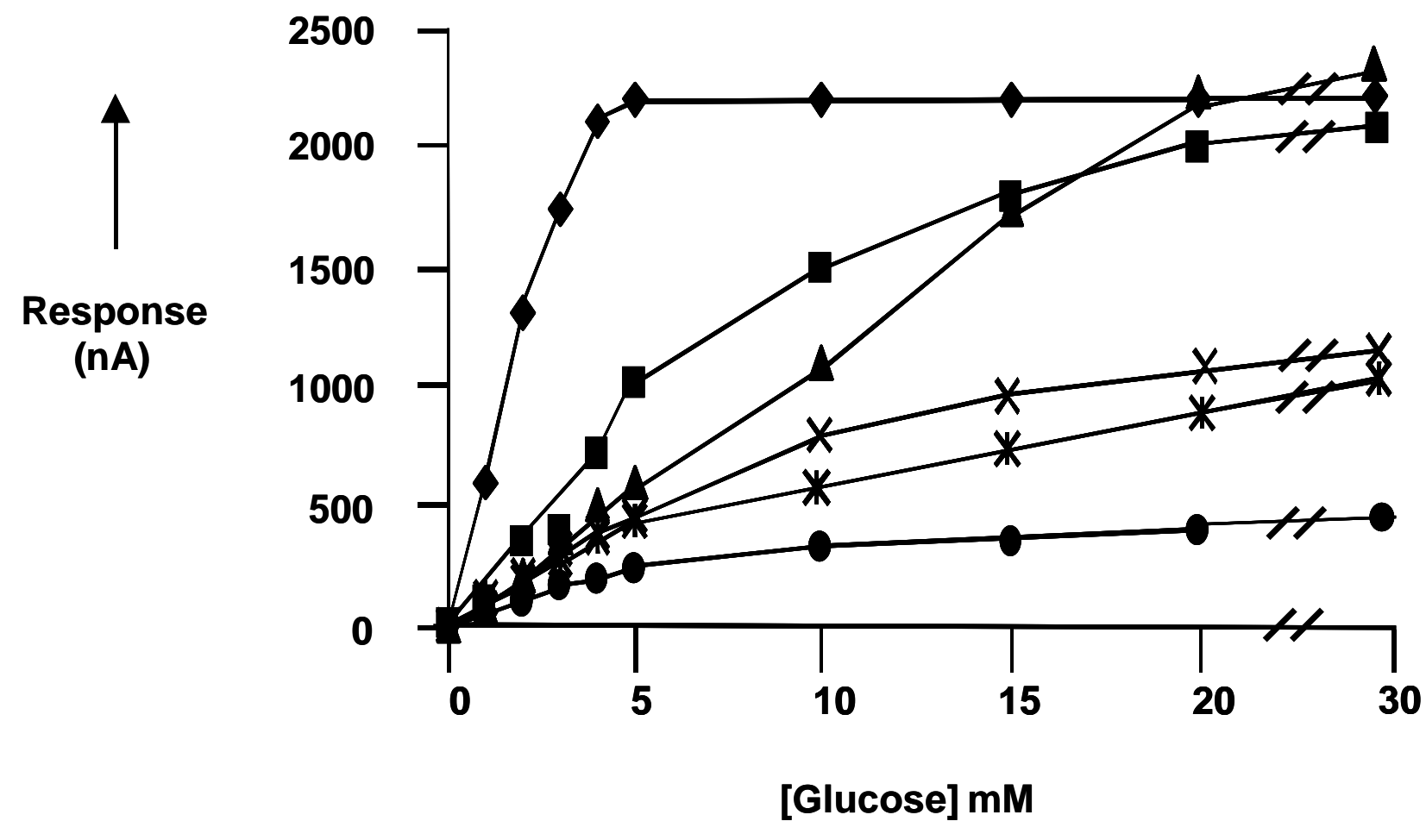




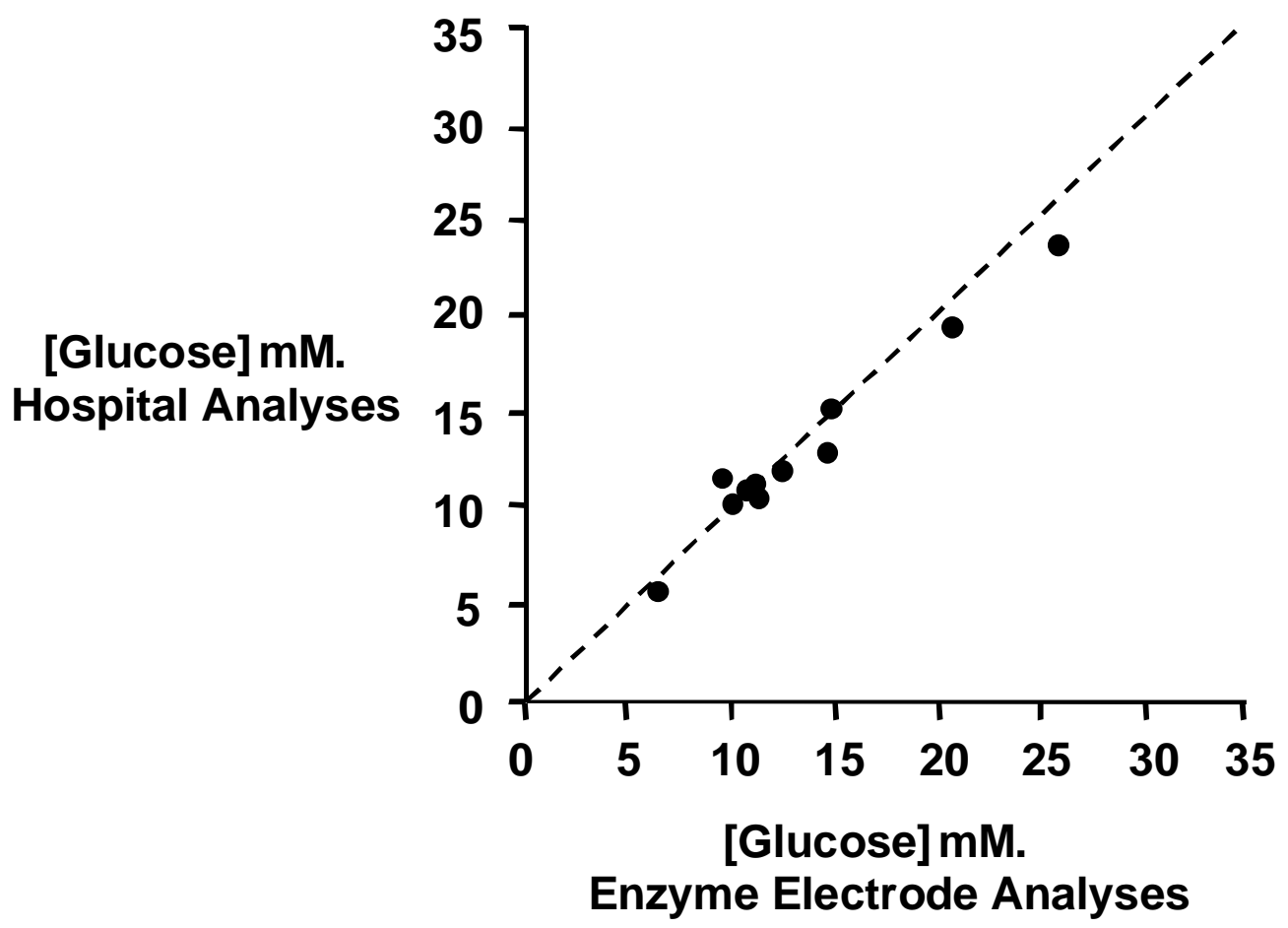



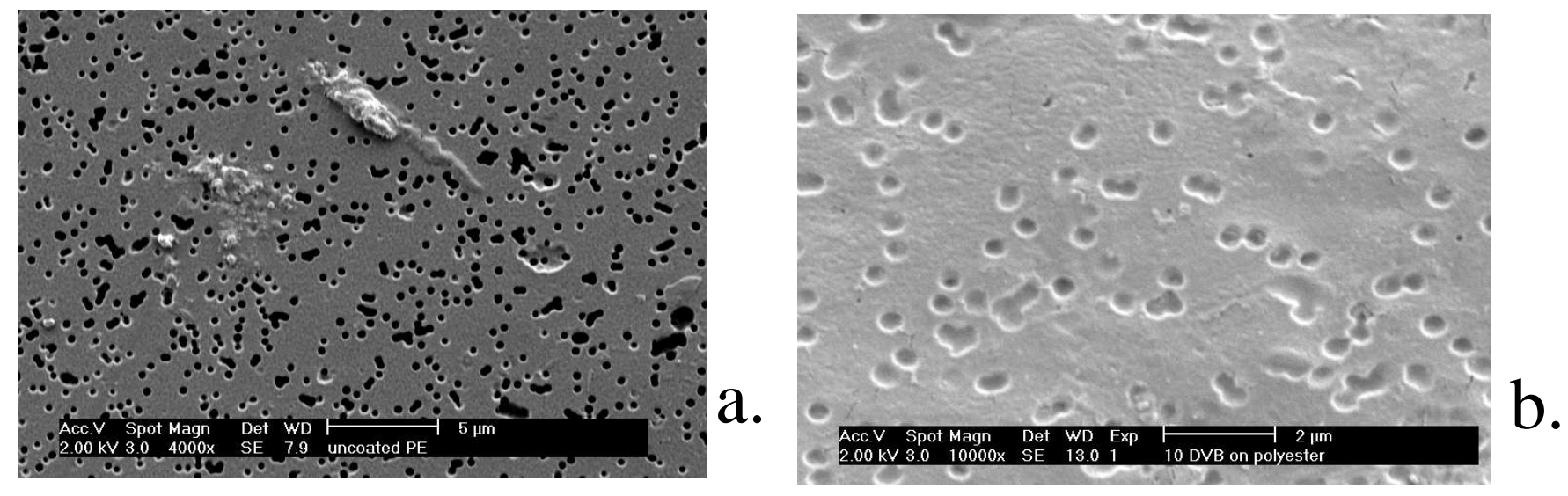